\title{
Association between mindfulness, social cognition, and social functioning: an exploratory and proof-of-concept analysis
}

\author{
R. Mediavilla²,3, A. De Diego ${ }^{1}$, A. Munoz-Sanjose ${ }^{1,2}$, A. Orosa ${ }^{1}$, M.P. Vidal-Villegas ${ }^{2}$, N. Fernandez ${ }^{1}$, A. Garcia ${ }^{1}$, G. Kollias ${ }^{1}$, J.M. Pastor ${ }^{1}$, C. Bayon ${ }^{1,2}$, S. Cebolla ${ }^{1}$, G.
} Lahera $^{4,5}$, A. Palao ${ }^{1,2}$, B. Rodriguez-Vega ${ }^{1,2}$, E. Roman ${ }^{1}$, P. Sanchez ${ }^{1}$, P. Garcia ${ }^{1}$, I. Gonzalez-Bocelo ${ }^{1}$, J.J. Rodriguez-Solano ${ }^{1}$, M.F. Bravo ${ }^{1,2}$

1La Paz University Hospital, Psychiatry- Clinical Psychology and Mental Health, Madrid, Spain.

2 Hospital La Paz Institute for Health Research (IdiPAZ), Psychiatry and Mental Health Group, Madrid, Spain.

3 National University of Distance Education UNED, Psychology, Madrid, Spain.

4University of Alcala, Faculty of Medicine. Psychiatry Department, Alcala de Henares, Spain.

5 CIBERSAM, Mental Health, Madrid, Spain.

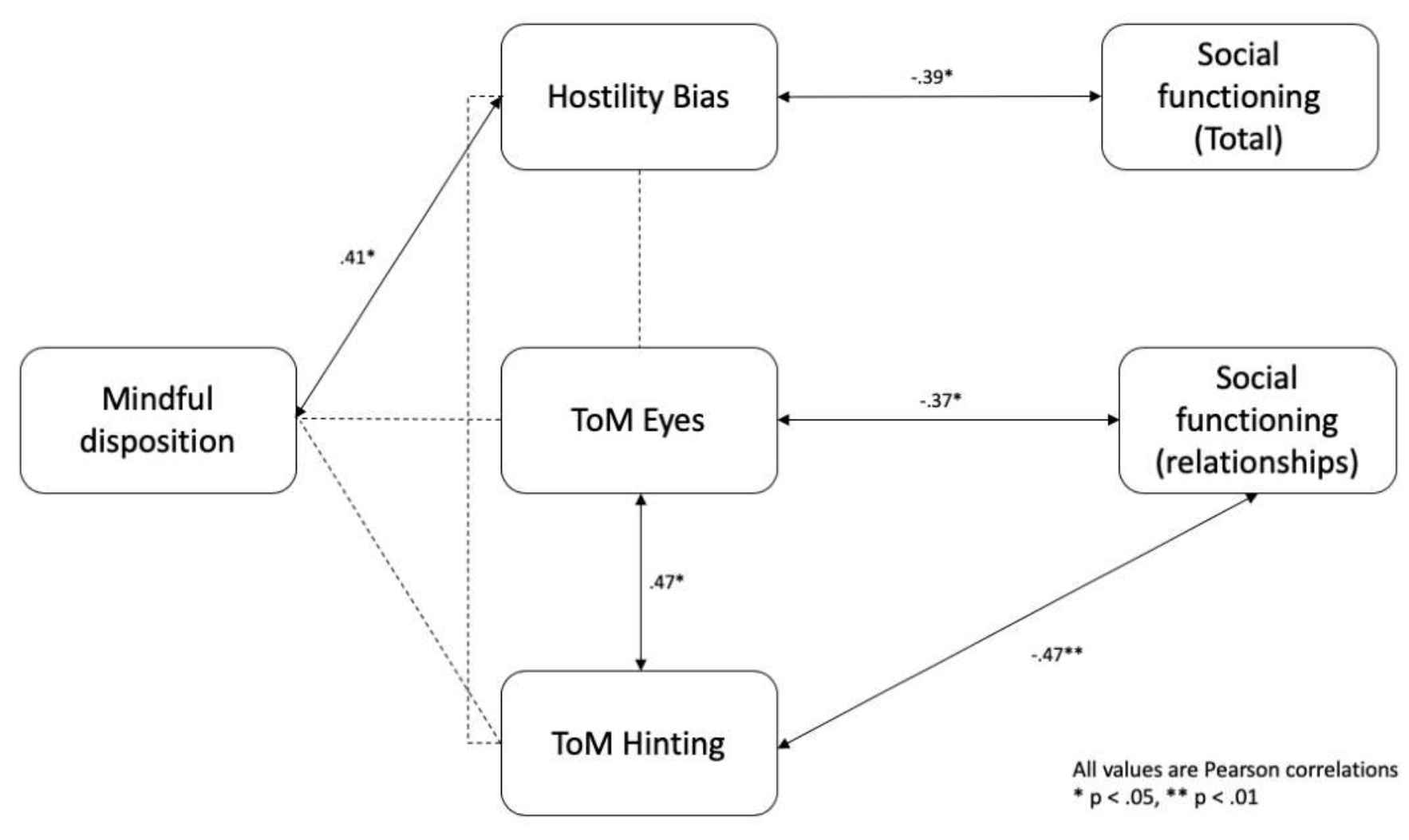

Background

Real-life outcomes are becoming more relevant as a measure of the effectiveness of interventions. People with schizophrenia spectrum disorders (SSD) often have difficulties engaging in daily activities and spending time with their peers, which are core elements of social functioning (Palumbo et al., 2015). Social cognition is a core predictor of social functioning (Green et al., 2015), but no mindfulness-based interventions (MBIs) targeting aspects of social cognition have been developed for people with psychosis.

\section{Objective}

To test associations between mindful disposition, social cognition, and social functioning, in a sample of people with SSD enrolled in a mindfulness-based social cognition training (SocialMIND) (see Table 1). Scan for additional material:
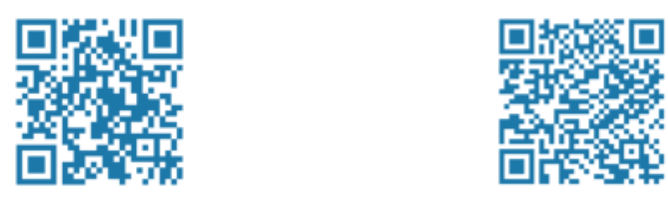

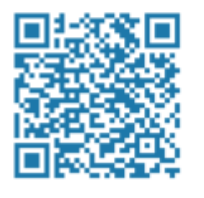

Material and methods

A sample of $\mathbf{3 0}$ participants with SSDs was included. Theory of mind was measured with the short version of the Hinting Task and with the Reading the Mind in the Eyes Test (RMET). Attributional Style was measured with the Attribution of Intentions and Hostility Questionnaire (AIHQ). Mindful Attention and Awareness Scale (MAAS) measured mindful disposition. Finally, social functioning was evaluated with Personal and Social Performance (PSP).

\section{Results and Conclusions}

Pearson correlations are shown in Figure 1. In keeping with previous reports, social cognition was moderately associated with social functioning (Couture et al., 2006). Hinting Task and RMET assess mental state attribution, and its association was expected (Browne et al., 2016); on the other hand, they were not associated with attributional style. Further, MAAS was linked to attributional style, but not with mental state attribution.

We hypothesized that an MBI should focus on attributional bias rather than theory of mind in order to improve social functioning.

\begin{abstract}
Welcome meditation ( $\left.3^{\prime}\right)$ Summary of previous session ( $\left(^{\prime}\right)$

Home-practice review $\left(5^{\prime}\right)$
\end{abstract}

Exposition (10')

Practices $\left(25^{\prime}\right)$

Exercises (20')

Summary and closure (10')

Home-practice presentation (5') 3-min guided meditation

Brief summary of the contents of session 4

Experience during informal (i.e., mindful walking) and formal (i.e., recordings of guided meditations) practices during the week. Focus on any difficult with training.

Self-compassion and loving kindness promote health and wellbeing. The importance of be aware of self-criticism and judging attitudes through oneself and others.

Soothing touch: Participants are encouraged to find a part of their body where a gentle touch is soothing.

Mindful movements: Participants perform simple exercises consisting of moving their arms up and down, or stretching their bodies, with the instruction of keeping full awareness.

Loving kindness: teachers include phrases that promotes a mental state of unselfish and unconditional kindness to all beings, especially oneself.

Emotion recognition: Participants are encouraged to identify basic emotions in a series of pictures. They also discuss how they feel and how would they react to each facial expression.

Attributional style: Teachers read some stories out loud. Participants are invited to think about characters' performance and to imagine how would they feel.

Teachers summarize today's session. Participants and teachers summarize in one short sentence what they have learned or how the session made them feel

Formal practices. Teachers give participants two new audio recordings (soothing touch and loving kindness).

Informal practices. Participants are told to practice mindful engagement in daily activities (driving, eating, having a shower...), self-care (enjoy a meal or a walk), self-compassion (kind inner talk) and kind gestures (i.e., to hold the door) during the week. 